\title{
Anaesthetic modality and post-surgical oncological outcomes for paediatric tumours: is there a link?
}

\author{
Shu Ying Lee ${ }^{1}$, MMed, Sanchalika Acharyya ${ }^{2}$, PhD, Ashley Wan Ling $\underline{\operatorname{Tan}^{3}}$, MBBS, Amos Hong Pheng $\underline{L o h}^{4}$, FRCS
}

\begin{abstract}
INTRODUCTION: Children with solid organ tumours often present for curative surgery. Even with the best surgical technique, micrometastases can occur. Preclinical studies support the postulation that neuraxial anaesthesia maintains the body's immune and inflammatory milieu against metastasis. However, human retrospective adult studies showed varying results, and no study has been done in children. We aimed to find out if intraoperative epidural, perioperative opioid and volatile dose are associated with relapse-free survival (RFS) in children with solid organ tumours.

METHODS: This is a retrospective cohort study of 126 children from a tertiary paediatric unit who were diagnosed with solid organ tumours (neuroblastoma, hepatoblastoma or sarcoma) over a 16-year period. RFS, stratified by tumour subtypes, was estimated using the Kaplan-Meier method. Adjusted hazard ratios (aHRs) were obtained from multivariable Cox regression models after taking potential covariates into account.

RESULTS: Of 126 children with solid organ tumours (51.6\% neuroblastoma, 34.9\% sarcoma and $13.5 \%$ hepatoblastoma), $53.2 \%$ received combined general anaesthesia (GA)/epidural. A total of $21(31.3 \%)$ and 20 (33.9\%) patients relapsed during the study period in the combined GA/epidural group and the GA alone group, respectively. Patients with sarcoma receiving combined GA/epidural had a clinically meaningful lower risk of relapse compared to patients receiving GA alone (aHR $0.51,95 \%$ confidence interval $0.14-1.79$ ), although this was not statistically significant.

CONCLUSION: Our study demonstrated some clinically meaningful associations, especially in paediatric sarcoma patients. Overall, however, there was no statistically significant association between epidural use and an improved RFS.
\end{abstract}

Keywords: anaesthesia, analgesia, cancer recurrence, paediatric, regional techniques

\section{INTRODUCTION}

Children with localised paediatric tumours often present for resection with curative intent. In Singapore, the overall childhood cancer age-standardised incidence rate (ASR) is 101.56 per million. ${ }^{(1)}$ Even with the best surgical technique, recurrence remains possible, as dormant tumour cells may already exist in distant sites and surgical resection may release tumour cells into circulation, leading to micrometastasis and residual disease. At present, the overall five-year survival rates of neuroblastoma, hepatoblastoma and sarcoma are $80 \%, 65 \%$ and $70 \%$, respectively. Perioperative immunological surveillance plays a crucial role in eradicating cancer cells and preventing disease progression of residual circulating cancer cells. ${ }^{(2)}$

The stress response during primary cancer surgery potentially creates conditions that are conducive to cancer cell survival and spread. ${ }^{(3)}$ Animal and adult studies have postulated three perioperative factors ${ }^{(4)}$ that could cause progression of minimal residual disease. First, surgery itself depresses innate immunity (especially natural killer [NK] cell activity $\left.{ }^{(5)}\right)$, reduces tumourrelated anti-angiogenic factors ${ }^{(6)}$ (e.g. angiostatin and endostatin), increases pro-angiogenic factors (e.g. vascular endothelial growth factor $\left.{ }^{(7)}\right)$, and releases growth factors promoting tumour growth. Second, anaesthetic agents (e.g. halothane, isoflurane and sevoflurane) impair immune functions, including those of neutrophils, macrophages, dendritic cells, T-cells and NK cells in mice ${ }^{(8)}$ and humans. ${ }^{(9)}$ Third, opioid analgesics inhibit cellular and humoral immune function in humans, ${ }^{(10)}$ while morphine promotes angiogenesis of human breast cancer cells in mice models. $^{(11)}$

In light of the above, anaesthetists have looked into modifiable perioperative factors that may help preserve the body's immunological milieu against tumour activity. Of particular interest is how neuraxial anaesthesia may reduce metastases by dampening the surgical stress response, sparing opioid and/or volatile requirements and providing potential direct anti-cancer and anti-inflammatory effects. ${ }^{(12)}$ In rodent studies, spinal blockade attenuated the development of lung and liver metastases. ${ }^{(13,14)}$ However, retrospective human studies done in adult tumours (including breast, prostate and colorectal cancers) have had nonconclusive results. ${ }^{(15-17)}$ Prospective randomised trials are currently ongoing, ${ }^{(4)}$ while secondary analyses of previously conducted trials ${ }^{(18)}$ have yielded poor-quality evidence that was insufficient to affect clinical decisions.

It is also not known if regional anaesthesia affects the body's immunological surveillance to different extents in different tumour types. Furthermore, no studies have investigated whether these postulations apply to paediatric tumours. The rarity of paediatric tumours presenting for surgery makes the design of prospective studies challenging. Furthermore, only some paediatric tumours are amenable to surgical resection, and 


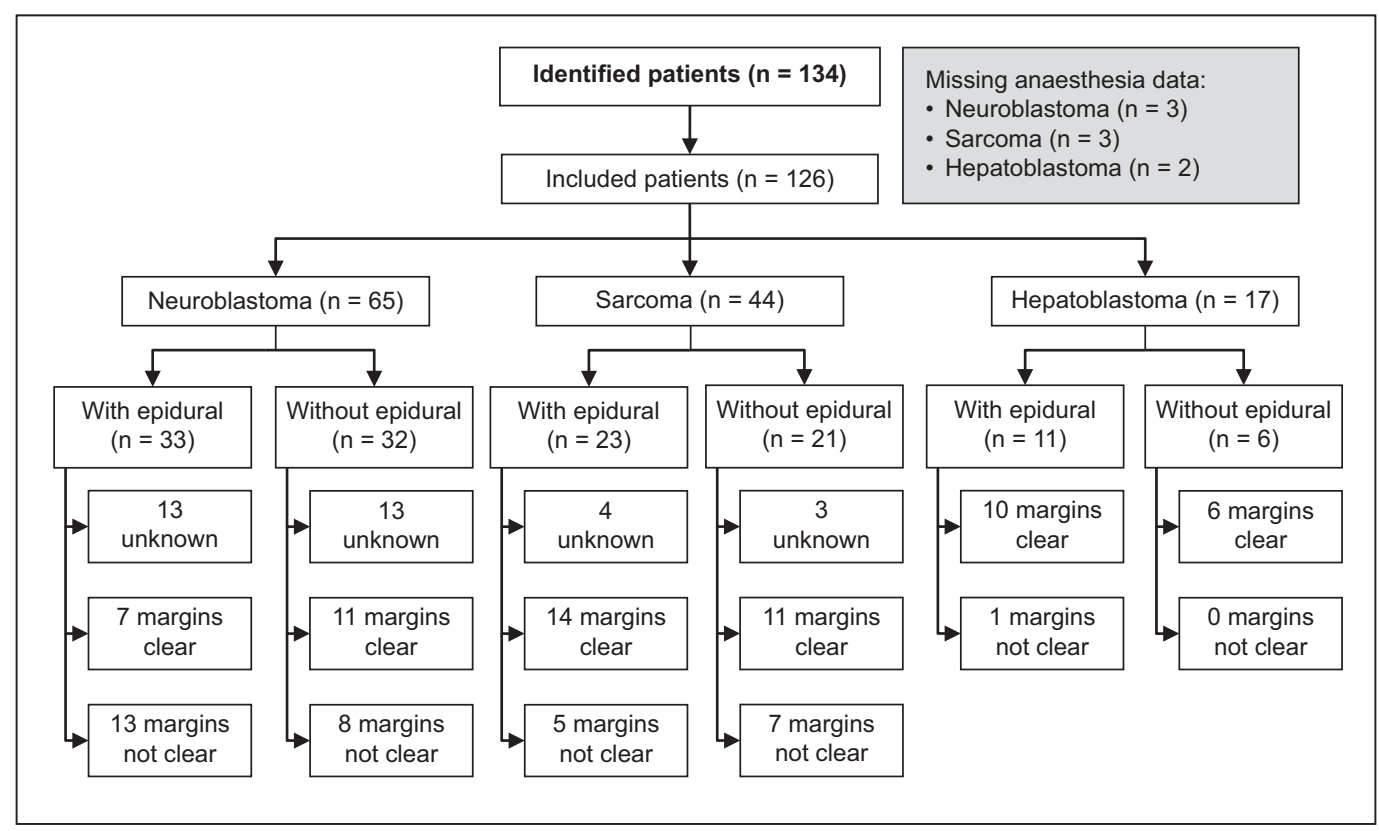

Fig. 1 STROBE flow diagram shows inclusion process for patients in the study.

only those localised to the trunk and lower limbs are suitable for epidural analgesia.

This study aimed to find out if intraoperative epidural analgesia, perioperative opioid use and total dose of volatile agent used are associated with relapse-free survival (RFS) of paediatric patients after primary tumour excision surgery, when stratified by tumour subtypes. Our hypothesis was that the use of combined general anaesthesia (GA) and epidural analgesia (combined GA/epidural) improves RFS in children with solid organ tumours undergoing primary tumour excision surgery. Our secondary hypothesis was that a reduced dose of volatile agent and perioperative opioid is associated with improved RFS.

\section{METHODS}

This study was approved by the SingHealth Centralised Institutional Review Board. Waiver of consent was given. All patients diagnosed with either neuroblastoma, hepatoblastoma or sarcoma, who underwent primary resection of their tumour at our tertiary paediatric unit from January 1997 to December 2012, were eligible for this retrospective study. The majority of the patients from the database with solid organ tumours had these three primary diagnoses. Moreover, these three tumour subtypes are mainly localised to the trunk and limbs, and are thus amenable to epidural analgesia. Excluded patients were those with previous resections (except for diagnostic biopsies) or factors that could predispose them to higher risk of mechanical dissemination, metastases or distant organ failure (such as known genetic risk factors and/or pre-surgical rupture or pathologic fractures).

Patients were identified from the Singapore Childhood Cancer Registry (SCCR) located in KK Women's and Children's Hospital, Singapore. This registry holds records of patient demographics, disease, treatment and oncologic outcomes, and follow-up data. At the point of data extraction on 31 March 2015, the registry held 3,227 records. As the only public tertiary children's hospital and main referral centre for paediatric cases, the SCCR represents the majority of our country's childhood tumour caseload. A total of 154 cases (76 neuroblastoma, 21 hepatoblastoma and 57 sarcoma cases) were identified from the database. Among these, 134 patients who were post-surgery for at least three years were included in our study (Fig. 1). This was to allow time for recurrence to take place.

Eligible patients were matched to the KK Paediatric Anaesthesia Audit Database to extract details of the primary resection surgery performed. Patient case notes were also traced to extract further details of anaesthetic agents used during the perioperative period of the primary surgery. We chose to look only at the primary resection surgery to concentrate on the effect of the anaesthetic technique used in that setting; it is also the most crucial time during which eradication of disseminated circulating tumour cells occurs, affecting the potential for subsequent remission. All patients were followed up after the primary surgery, regardless of subsequent treatment. An event was recorded as long as relapse occurred during the follow-up period.

Anaesthetic data collected included the following: type and duration (in minimum alveolar concentration [MAC] hours) of volatile agent used; level and duration of epidural analgesia; amount of epidural and adjuvants used (including bolus dose and intraoperative/postoperative infusions); and total dose of intravenous opioids used (intraoperative and postoperative).

All statistical analyses were performed using $\mathrm{R}$ 3.0.1 (R Foundation for Statistical Computing, Vienna, Austria). Patient demographics and clinical characteristics were compared between the patients who received GA alone and those who received combined $\mathrm{GA} /$ epidural, using chi-square test and $t$-tests for categorical and continuous variables, respectively. Three-year relapse-free and death-free rates were estimated and compared between these two groups of patients for each tumour subtype.

The primary outcome of interest was RFS in years, which was defined as the time interval between the date of the primary resection and the date of the first relapse recorded. Patients 
who died of the disease or any other cause were censored on their date of death. Those who were relapse-free and alive were censored at their last follow-up date. The secondary outcome of interest was overall survival (OS) in years, defined as the time elapsed from the date of resection to the date of death from any cause.

RFS and OS, stratified by tumour type, were estimated using the Kaplan-Meier method. Survival curves of patients who received GA alone and those who received combined GA/ epidural were compared using the log-rank test. Cox proportional hazards models were fitted, separately for each tumour subtype, to estimate hazard ratios (HRs) with $95 \%$ confidence intervals (Cl) and thereby, examine the association between epidural analgesia use and RFS. In subsequent multivariable Cox regression models, these HRs were adjusted by taking into account variables that were considered potential confounding factors of the association being studied (i.e. age, gender, tumour stage and spread of tumour). A p-value $<0.05$ was used to indicate statistical significance. Similar analyses were carried out to study the association of RFS with total perioperative doses of morphine (in $\mathrm{mg} / \mathrm{kg}$ ) and fentanyl (in mcg/ $\mathrm{kg}$ ) and total dose of volatile agent (in MAC hours) used. A subgroup analysis was also done for the patients with complete histological excision (i.e. margins clear), a surgical factor that is associated with lower recurrence risk. All analyses were planned a priori.

\section{RESULTS}

A total of 134 subjects were identified as eligible, but eight were excluded because we could not trace their anaesthetic charts. Of the 126 included subjects (61 female, 65 male) with solid organ tumours, $65(51.6 \%)$ were diagnosed with neuroblastoma, $44(34.9 \%)$ with sarcoma and 17 (13.5\%) with hepatoblastoma. The median age at primary surgical resection was 3.5 (range $0-16.1$ ) years. Tumour stage at resection was significantly different between the GA only and combined GA/epidural groups, and the latter had a higher proportion of Stage 3 and 4 tumours of the neuroblastoma and hepatoblastoma subtypes. Median follow-up time was 2.14 years. $59(46.8 \%)$ of the 126 children achieved complete resection (i.e. margins clear) on histology (Fig. 1).

The demographic and clinical characteristics of the study population are illustrated in Table I, including factors used in tumour risk stratification, namely age, clinical features of tumour spread and stage (which was determined by imaging and biopsies). Locally, we use the SIOPEL (Societe Internationale Oncologie Pediatrique Europe - Liver) risk classification for hepatoblastomas and COG (Children's Oncology Group) staging guidelines for neuroblastomas and sarcomas.

Table I summarises the intraoperative epidural analgesia, perioperative volatile agents and intravenous opioid agents used during the primary resection surgery. Intraoperative epidural analgesia was given to 67 (53.2\%) children. The 16 -year period was divided into two eight-year periods (1997-2004 and 2005-2012), with 37 children's surgeries in the former time period and 89 in the latter time period. The proportion of children who received GA/epidural did not change significantly (59.5\% in 1997-2004 vs. 50.6\% in 2005-2012). The choice of epidural agent was bupivacaine in all except one child, for whom ropivacaine was used. The mean amounts of bupivacaine used were $15.9 \mathrm{mg} / \mathrm{kg}, 19.6 \mathrm{mg} / \mathrm{kg}$ and $11.7 \mathrm{mg} / \mathrm{kg}$ for children with neuroblastoma, hepatoblastoma and sarcoma, respectively. Both fentanyl and clonidine were used as adjuvant epidurals, although clonidine was used only in two children. The adjuvants were added to the epidural local analgesic infusion in concentrations of $2 \mathrm{mcg} / \mathrm{mL}$ and $1 \mathrm{mcg} / \mathrm{mL}$, respectively. Children with neuroblastoma and hepatoblastoma received largely thoracic epidurals $(69.7 \%$ and $100 \%$, respectively), whereas those with sarcoma received mostly lumbar epidurals (87.0\%).

Of the children with sarcomas, 30 children had sarcomas located on a limb, while 14 sarcomas were located on the trunk (one abdomen and 13 pelvis). Half of the children with limb sarcomas received epidurals and half did not (two children who did not have an epidural received nerve blocks). In children with trunk sarcomas, 8 (57.1\%) received an epidural and 6 (42.9\%) did not, with one child who did not have an epidural receiving an ilioinguinal nerve block. Epidural catheters were kept for a maximal duration of six days. Volatile agents used included desflurane, sevoflurane and isoflurane. There was no statistically significant difference in the amounts of volatile agent used (in MAC hours) when analysed by tumour subtypes. Intravenous opioids used included morphine and fentanyl. Although the difference was not statistically significant, total intravenous opioid exposure, calculated per kilogramme of body weight, was generally lower in the combined GA/epidural group for all three tumour subtypes, demonstrating the opioid-sparing effect of epidural analgesia. However, no similar volatile-sparing effect was demonstrated in the combined GA/epidural groups across the tumour subtypes.

During the study follow-up period, a total of 41 (32.5\%) children had a relapse and $39(31.0 \%)$ children died of any cause. Of the relapsed patients, 21 (31.3\%) patients and 20 $(33.9 \%)$ patients were in the combined GA/epidural and the GA alone groups, respectively. RFS at three years was $63.7 \%$ (95\% $\mathrm{Cl} 55.2 \%-73.6 \%)$ for the overall population, $64.1 \%(95 \% \mathrm{Cl}$ $52.5 \%-78.1 \%$ ) for those with combined GA/epidural, and $63.3 \%$ (95\% Cl 51.4\%-78.0\%) for those with GA alone. OS at three years was $66.3 \%$ (95\% Cl $57.7 \%-76.1 \%$ ) for the overall population, $68.6 \%(95 \% \mathrm{Cl} 57.2 \%-82.3 \%)$ for those with combined GA/ epidural, and $63.9 \%$ (95\% Cl 51.9\%-78.9\%) for those with GA alone. Absolute survival rates by tumour subtypes are summarised in Table I.

RFS was not significantly different between those receiving combined GA/epidural and those receiving GA alone within any of the tumour subtypes (Fig. 2). HRs, when adjusted for age, gender, tumour stage and the presence of spread of disease, suggested no benefit in survival endpoint among children with neuroblastoma. Although it was not statistically significant, the children with sarcoma who received combined GA/epidural had half the risk of relapse when compared to those who received only GA (adjusted HR [aHR] 0.51, 95\% Cl 0.14-1.79; Fig. 2 \& Table II). 
Original Article

Table I. Summary of patient demographics, clinical characteristics and anaesthesia/analgesia used during primary surgery.

\begin{tabular}{|c|c|c|c|c|c|c|c|c|c|c|}
\hline \multirow[t]{3}{*}{ Parameter } & \multicolumn{9}{|c|}{ No. (\%) } & \multirow[t]{3}{*}{ p-value* } \\
\hline & \multicolumn{3}{|c|}{ Neuroblastoma $(n=65)$} & \multicolumn{3}{|c|}{ Hepatoblastoma $(n=17)$} & \multicolumn{3}{|c|}{ Sarcoma $(n=44)$} & \\
\hline & $\begin{array}{l}\text { GA only } \\
(\mathrm{n}=32)\end{array}$ & $\begin{array}{l}\text { Combined GA/ } \\
\text { epidural }(n=33)\end{array}$ & p-value & $\begin{array}{l}\text { GA only } \\
(n=6)\end{array}$ & $\begin{array}{l}\text { Combined GA/ } \\
\text { epidural }(n=11)\end{array}$ & p-value & $\begin{array}{l}\text { GA only } \\
(n=21)\end{array}$ & $\begin{array}{l}\text { Combined GA/ } \\
\text { epidural }(n=23)\end{array}$ & p-value & \\
\hline $\mathrm{Age}^{\dagger}(\mathrm{yr})$ & $3.2 \pm 2.6$ & $3.5 \pm 2.4$ & 0.571 & $1.1 \pm 0.4$ & $2.1 \pm 1.6$ & 0.116 & $8.5 \pm 5.3$ & $9.9 \pm 4.1$ & 0.292 & \\
\hline \multicolumn{11}{|l|}{ Gender } \\
\hline Female & $19(59.4)$ & $12(36.4)$ & 0.108 & $2(33.3)$ & $7(63.6)$ & 0.492 & $7(33.3)$ & $14(60.9)$ & 0.127 & \\
\hline Male & $13(40.6)$ & $21(63.6)$ & & $4(66.7)$ & $4(36.4)$ & & $14(66.7)$ & $9(39.1)$ & & \\
\hline \multicolumn{11}{|l|}{ Ethnicity } \\
\hline Chinese & $22(68.8)$ & $24(72.7)$ & 0.386 & $4(66.7)$ & $7(63.6)$ & 0.402 & $17(81.0)$ & $18(78.3)$ & 0.029 & \\
\hline Malay & $5(15.6)$ & $3(9.1)$ & & $0(0)$ & $1(9.1)$ & & $4(19.0)$ & $0(0)$ & & \\
\hline Indian & $4(12.5)$ & $2(6.1)$ & & $0(0)$ & $2(18.2)$ & & $0(0)$ & $1(4.3)$ & & \\
\hline Other & $1(3.1)$ & $4(12.1)$ & & $2(33.3)$ & $1(9.1)$ & & $0(0)$ & $4(17.4)$ & & \\
\hline Localised tumour & $13(40.6)$ & $12(36.4)$ & 0.922 & $5(83.3)$ & $9(81.8)$ & 0.999 & $17(80.9)$ & $18(78.3)$ & 0.999 & \\
\hline \multicolumn{11}{|l|}{ Tumour stage } \\
\hline 1 & $5(16.1)$ & $1(3.0)$ & 0.015 & $0(0)$ & $4(36.4)$ & 0.025 & $8(40.0)$ & $3(13.6)$ & 0.132 & \\
\hline 2 & $4(12.9)$ & $0(0)$ & & $5(83.3)$ & $1(9.1)$ & & $11(55.0)$ & $14(63.6)$ & & \\
\hline 3 & $4(12.9)$ & $11(33.3)$ & & $0(0)$ & $3(27.3)$ & & $1(5.0)$ & $4(18.2)$ & & \\
\hline 4 & $18(58.1)$ & $21(63.6)$ & & $1(16.7)$ & $3(27.3)$ & & $0(0)$ & $1(4.5)$ & & \\
\hline Epidural & NA & & & NA & & & NA & & & \\
\hline Type & & & & & & & & & & $<0.001$ \\
\hline Thoracic & & $23(69.7)$ & & & $11(100.0)$ & & & $0(0)$ & & \\
\hline Lumbar & & $3(9.1)$ & & & $0(0)$ & & & $20(87.0)$ & & \\
\hline Caudal & & $0(0)$ & & & $0(0)$ & & & $2(8.7)$ & & \\
\hline Thoracic and lumbar & & $5(15.2)$ & & & $0(0)$ & & & $0(0)$ & & \\
\hline Unknown & & $2(6.1)$ & & & $0(0)$ & & & $1(4.3)$ & & \\
\hline Duration (day) & & & & & & & & & & 0.103 \\
\hline 0 to $<1$ & & $0(0)$ & & & $0(0)$ & & & $1(4.8)$ & & \\
\hline 1 to $<2$ & & $1(3.6)$ & & & $2(20.0)$ & & & $0(0)$ & & \\
\hline 2 to $<3$ & & $10(35.7)$ & & & $2(20.0)$ & & & $8(38.1)$ & & \\
\hline 3 to $<4$ & & $5(17.9)$ & & & $2(20.0)$ & & & $9(42.9)$ & & \\
\hline 4 to $<5$ & & $9(32.1)$ & & & $4(40.0)$ & & & $3(14.3)$ & & \\
\hline 5 to $<6$ & & $3(10.7)$ & & & $0(0)$ & & & $0(0)$ & & \\
\hline Total LA bupivacaine ${ }^{\dagger}(\mathrm{mg} / \mathrm{kg})$ & & $15.9 \pm 9.0$ & & & $19.6 \pm 7.6$ & & & $11.7 \pm 6.2$ & & 0.058 \\
\hline Adjuvant fentanyl $\mathrm{l}^{+}(\mathrm{mcg} / \mathrm{kg})$ & & $25.3 \pm 14.1$ & & & $34.1 \pm 14.9$ & & & $24.1 \pm 12.6$ & & 0.23 \\
\hline
\end{tabular}




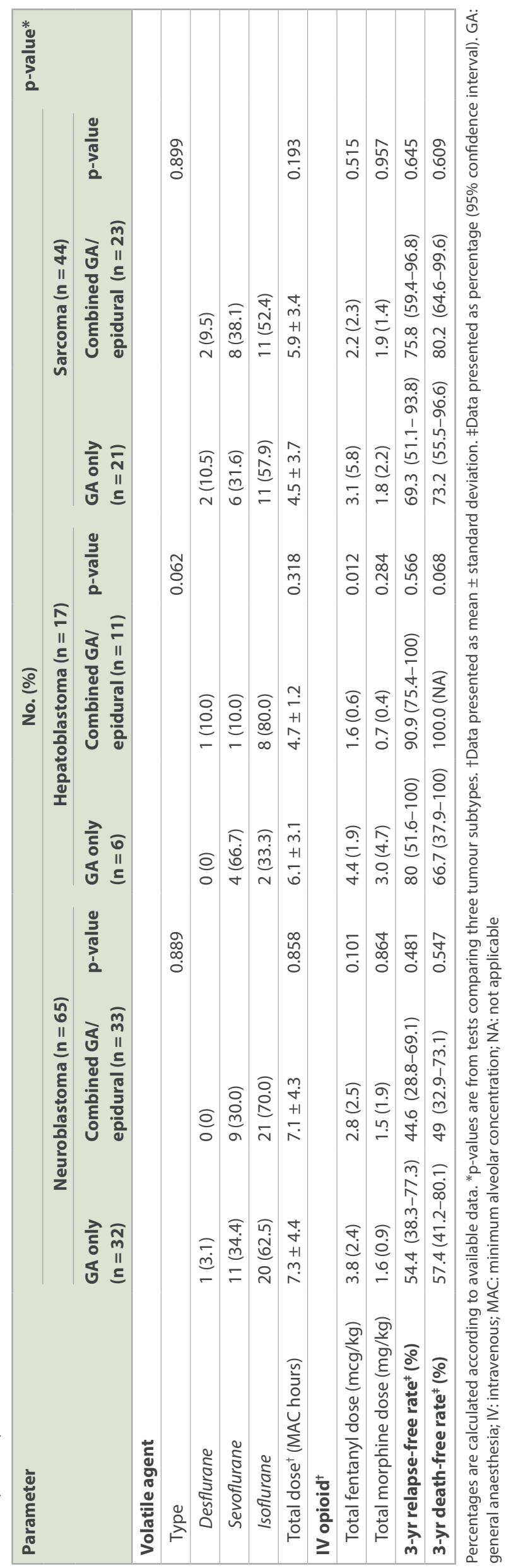

This result was the same for children with sarcoma who had complete histological excision (Fig. 3 \& Table II).

We also analysed separately the total perioperative doses of morphine (in $\mathrm{mg} / \mathrm{kg}$ ), fentanyl (in $\mathrm{mcg} / \mathrm{kg}$ ) and volatile agent (in MAC hours) used in relation to RFS (Table II). None of the aHRs for total dose of perioperative morphine reached statistical significance. The aHR for total perioperative fentanyl dose was close to 1 for both children with neuroblastoma and sarcoma. However, a significantly lower risk of relapse was demonstrated for total dose of volatile agent in children with sarcoma (aHR $0.67,95 \% \mathrm{Cl} 0.49-0.92)$. Interestingly, children with sarcoma and neuroblastoma who received isoflurane had a higher hazard of relapse (aHR 1.48, 95\% Cl 0.39-5.63, $\mathrm{p}=0.485$ and aHR 2.44, $95 \% \mathrm{Cl} 0.98-6.10, \mathrm{p}=0.093$, respectively), when compared to those who received sevoflurane, although the effect did not reach statistical significance in both groups.

OS was similar between the children who received and those who did not receive epidural analgesia among the included children as well as within the subgroup of children with complete histological excision.

\section{DISCUSSION}

While GA and opioids have been implicated in compromising the body's tumour immunological surveillance, the immunomodulative effects of surgical trauma and pain also cannot be ignored, ${ }^{(5)}$ leaving the anaesthetist in search of better anaesthesia and analgesia modalities. Paediatric patients rarely tolerate any surgery without GA. However, for paediatric tumour resections involving the trunk or limbs, the presence of a working epidural can potentially reduce the total dose of opioids needed for pain relief. The sympathetic blockade provided by neuraxial anaesthesia also attenuates the surgical stress response and its consequent immunosuppressive effect. In addition, local anaesthetic drugs used with the epidural technique may positively influence the patient's immune response. ${ }^{(19)}$

Our results did not support our hypothesis that epidural use improves RFS with sufficient statistical significance. This is contradictory to the in vitro, ${ }^{(20)}$ animal ${ }^{(21)}$ and some observational human studies ${ }^{(22-24)}$ that postulated a substantial reduction in cancer recurrence with epidural use. However, a clinically meaningful lower risk of relapse was observed in children with sarcoma who received combined GA/epidural as compared to GA alone. RFS was not significantly lower in children with neuroblastoma and hepatoblastoma who received combined GA/epidural, even though a significantly greater proportion of them were operated on at a higher tumour stage (Stages 3 and 4).

In adult studies on abdominal cancers, negative results have also been reported in a prospective randomised controlled clinical trial with long-term follow-up data, in which patients were randomly assigned to receive GA with or without epidural block for at least three postoperative days. The study found that RFS was similar in both the epidural and non-epidural groups. ${ }^{(25)}$ Our study also converged with the recent Cochrane analysis, ${ }^{(18)}$ which found no advantage to the use of combined GA/epidural over GA alone in terms of progression-free survival (PFS). Our 
Table II. Hazard ratios (HR) with confidence interval estimated using Cox proportional hazard regression models.

\begin{tabular}{|c|c|c|c|c|}
\hline \multirow[t]{3}{*}{ Parameter } & \multicolumn{4}{|c|}{ HR $(95 \% \mathrm{Cl})$} \\
\hline & \multicolumn{2}{|c|}{ All patients $(n=126)$} & \multicolumn{2}{|c|}{ Patients with complete histological excision $(n=59)$} \\
\hline & Univariate HR & Multivariable $\mathrm{HR}^{*}$ & Univariate HR & Multivariable $\mathrm{HR}^{*}$ \\
\hline \multicolumn{5}{|l|}{ Epidural use } \\
\hline Neuroblastoma & $1.14(0.53-2.46)$ & $0.84(0.37-1.92)$ & $1.01(0.18-5.52)$ & $0.85(0.12-6.27)$ \\
\hline Hepatoblastoma & $0.41(0.03-6.53)$ & $\mathrm{NE}$ & $0.45(0.03-7.18)$ & $\mathrm{NE}$ \\
\hline Sarcoma & $0.76(0.25-2.26)$ & $0.51(0.14-1.79)$ & $0.69(0.17-2.80)$ & $0.56(0.11-2.91)$ \\
\hline \multicolumn{5}{|c|}{ Total perioperative morphine dose $(\mathrm{mg} / \mathrm{kg})$} \\
\hline Neuroblastoma & $1.19(0.88-1.62)$ & $1.11(0.72-1.73)$ & $2.57(0.70-9.38)$ & $11.40(0.40-322.86)$ \\
\hline Hepatoblastoma & $0.73(0.12-4.41)$ & $\mathrm{NE}$ & $0.73(0.12-4.41)$ & $\mathrm{NE}$ \\
\hline Sarcoma & $1.06(0.77-1.46)$ & $0.91(0.56-1.49)$ & $0.82(0.48-1.39)$ & $0.64(0.28-1.47)$ \\
\hline \multicolumn{5}{|c|}{ Total perioperative fentanyl dose $(\mathrm{mcg} / \mathbf{k g})$} \\
\hline Neuroblastoma & $0.99(0.97-1.02)$ & $0.98(0.96-1.01)$ & $0.99(0.94-1.06)$ & $0.97(0.92-1.03)$ \\
\hline Hepatoblastoma & $1.01(0.94-1.08)$ & $\mathrm{NE}$ & $1.02(0.95-1.09)$ & $\mathrm{NE}$ \\
\hline Sarcoma & $0.97(0.92-1.03)$ & $0.96(0.90-1.02)$ & $1.01(0.95-1.07)$ & $1.02(0.92-1.12)$ \\
\hline \multicolumn{5}{|c|}{ Total volatile dose (MAC hours) } \\
\hline Neuroblastoma & $0.96(0.87-1.05)$ & $0.95(0.86-1.06)$ & $0.88(0.69-1.12)$ & $0.92(0.75-1.14)$ \\
\hline Hepatoblastoma & $0.26(0.05-1.44)$ & $\mathrm{NE}$ & $0.26(0.05-1.46)$ & $\mathrm{NE}$ \\
\hline Sarcoma & $0.79(0.63-1.02)$ & $0.67(0.49-0.92)$ & $0.81(0.60-1.09)$ & $0.87(0.63-1.20)$ \\
\hline
\end{tabular}

*Multivariable models were adjusted for age, gender, tumour stage and the presence of localised disease. Cl: confidence interval; MAC: minimum alveolar concentration; NE: not estimable due to small sample size
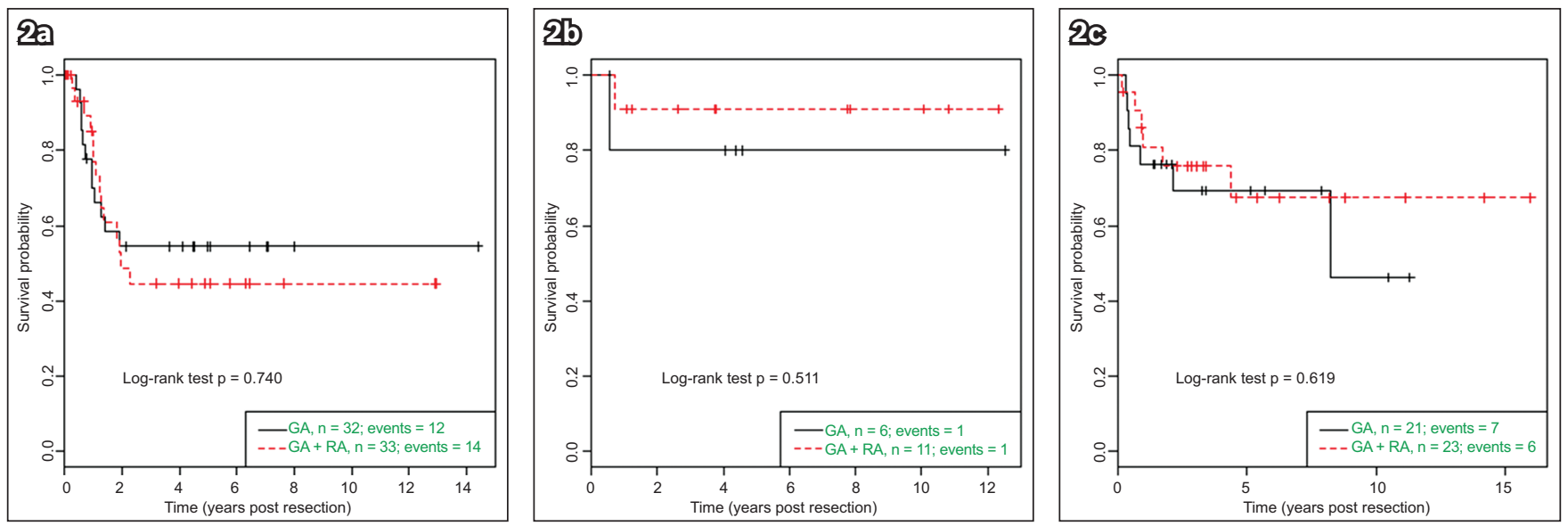

Fig. 2 Kaplan-Meier survival curves show relapse-free survival in patients with (a) neuroblastoma; (b) hepatoblastoma; and (c) sarcoma, by epidural use.
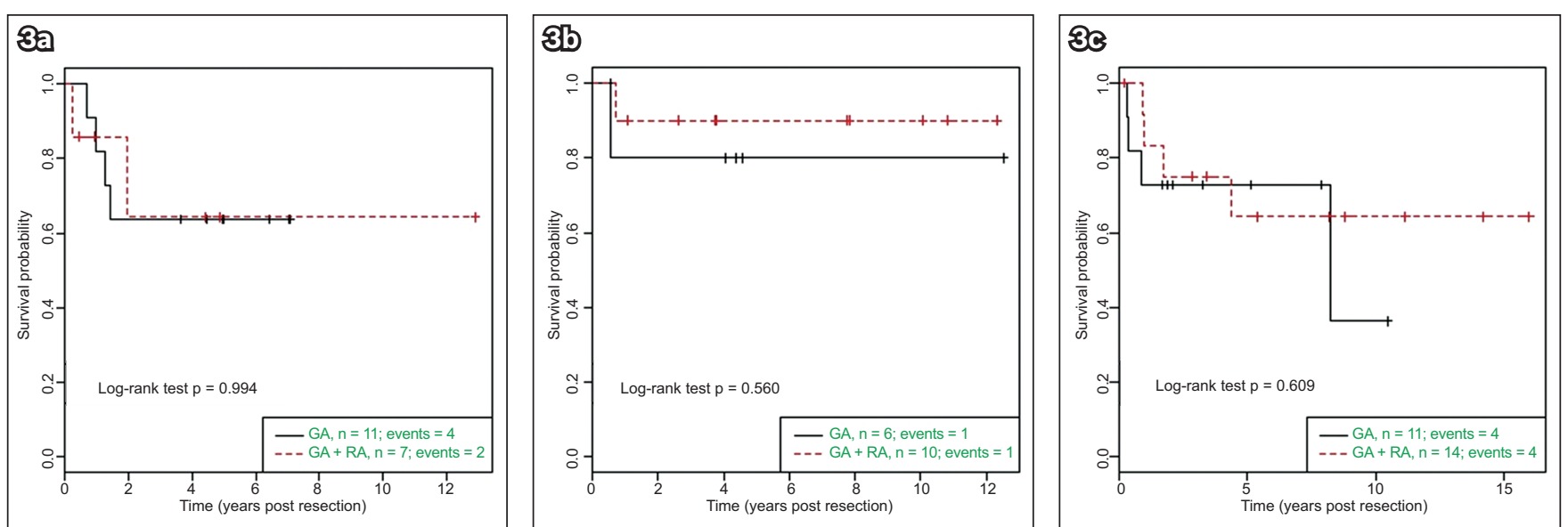

Fig. 3 Kaplan-Meier survival curves show relapse-free survival within the subgroup of patients with complete histological excision of (a) neuroblastoma; (b) hepatoblastoma; and (c) sarcoma, by epidural use. 
outcome measure of RFS is comparable to the Cochrane PFS outcome (HR 0.88, 95\% Cl 0.56-1.38).

More recently, an updated meta-analysis ${ }^{(26)}$ found a significant association between neuraxial anaesthesia and improved RFS (HR 0.846, 95\% Cl 0.718-0.998, $\mathrm{p}=0.047$ ) compared to when GA is used alone, with five out of 19 studies showing positive relationships between neuraxial anaesthesia and improved RFS. However, they were all retrospective studies involving adult tumours (e.g. breast, prostate, ovarian, gastro-oesophageal and laryngeal/pharyngeal).

The effect of opioids on cancer recurrence remains controversial to date. It appears that the concentration and duration of treatment has a role to play in determining the outcome of cell proliferation versus cell death. Some studies suggest that cell proliferating effects occur at low concentrations of opioids, while growth inhibiting effects occur at high concentrations or with chronic opioid treatment. ${ }^{(27)}$ In several human cancers, the $\mu$-opioid receptor is overexpressed, leading to the promotion of tumour growth and metastasis. However, no associations were found for all three tumour subtypes in our paediatric population. Differences in the cancer cell types in children as compared to adult tumours could potentially account for the differences in response to morphine administration. ${ }^{(28)}$ Neuroblastomas and hepatoblastomas are primitive, small, blue, round cell tumours that usually occur in younger age groups, whereas sarcomas fall into a separate category of mesenchymal tumours occurring in adolescents. Studies in this area are limited to a study by Olbrecht et al, ${ }^{(29)}$ which used immunohistochemistry to illustrate differences in $\mu$-opioid receptor behaviour in paediatric tumours, as compared to adult tumours.

Besides the heterogeneity in animal models, tumour subtypes and doses or routes of administration, different types of opioids also exhibit different effects on NK cell function. Fentanyl has been reported to highly increase NK cell cytotoxicity, ${ }^{(30)}$ while remifentanil has not been shown to impair NK cell function. ${ }^{(31)}$ In our study, besides intravenous administration during surgery, fentanyl was also the main adjuvant used in epidural infusions. Multivariable analysis of the total perioperative dose of fentanyl used in our study did not reveal any significant association with risk of relapse in all three tumour subtypes. This is in keeping with the findings of Owusu-Agyemang et al, ${ }^{(32)}$ who found no statistically significant association between opioid consumption and recurrence-free survival or OS in children with peritoneal carcinomatosis undergoing cytoreductive surgery with hyperthermic intraperitoneal chemotherapy.

All but one patient received bupivacaine in the epidural infusion, owing to its long duration of action and relatively low cost and long history of use in our institution. Several mechanisms, ${ }^{(33-35)}$ including tumour suppressor gene activation, ${ }^{(33)}$ inhibition of tumour cell migration and anti-proliferative effects, ${ }^{(35)}$ have been proposed to explain the anti-cancer effects of systemic amide-type local anaesthetic agents. Cytotoxic effects have been demonstrated to different extents based on the agents' lipophilicity and potency. ${ }^{(35)}$ However, most were in vitro studies on varying cell types and involved local anaesthetic doses that are above toxic thresholds. In our study, a lower hazard of relapse was indeed seen in children with sarcoma who had received combined GA/epidural, although this was not statistically significant.

Alpha 2-adrenoceptors are expressed in human breast cancer and non-cancer cell lines, and have been found to increase cell proliferation. ${ }^{(36,37)}$ Clonidine, with its alpha 2-adrenoceptor agonistic effect, has been shown to increase tumour growth and modulate NK cell activity in rodent studies. ${ }^{(38)}$ However, this relationship could not be investigated in our study, as clonidine was used as an epidural adjuvant in only two children.

In the present study, the total dose of volatile agent used was associated with lower risk of relapse in patients with sarcoma. This result appears to contradict theories about the oxidative stress effects of inhalational anaesthetic agents. ${ }^{(39)}$ Isoflurane use was found to be clinically associated with a higher hazard of relapse in the neuroblastoma subgroup, when compared with sevoflurane. However, given the shift toward the use of desflurane and sevoflurane over the study period, the observed effect could have been confounded by improvements in surgical and anaesthetic techniques over the years.

This study was not without limitations. As a retrospective study, it had the potential flaws associated with such studies. ${ }^{(4)}$ Heterogeneity of population and tumour subtypes resulted in non-conclusive results in previous studies. ${ }^{(18,26)}$ In our study, we took steps to control for confounders by stratifying the population according to tumour subtypes before analysis, adjusting for patient demographics, tumour stage and spread in multivariable regression model, and subanalysing the groups based on completeness of histological resection. In spite of this, it is difficult to isolate other confounders in the context of a multifactorial perioperative environment. Surgical and anaesthetic techniques would certainly have evolved over the years and may have impacted oncologic outcomes. Other perioperative confounding factors that were not captured on our data collection form but may affect cancer recurrence include adjuvant chemotherapy/radiotherapy, allogeneic blood transfusions, hypothermia and the use of beta-blocking agents.

Methodological constraints, such as the small sample size due to the rarity of paediatric solid organ tumours, also limited the interpretation of potential positive effects from our study. Although it would be impossible to retrospectively establish a causal relationship between techniques and outcomes, it is equally challenging to perform a prospective study on this topic due to the rarity of the disease. As a first preliminary effort to examine this aspect of paediatric tumours, our study has served to evaluate and identify the factors associated with better oncological outcomes.

In conclusion, this retrospective study demonstrated some clinically meaningful associations, especially in paediatric sarcoma patients. Overall, there was no statistically significant association between epidural use and an improved RFS. Further studies may explore the monitoring of inflammatory and immunological markers, including immune cell counts and activity, to have a better understanding of the possible mechanisms by which anaesthetics impact cancer recurrence. A collaborative multicentre approach to future prospective randomised studies might be the solution to achieving adequate sample sizes, so that 
the exact role of epidural use in cancer recurrence after primary resection surgery may be determined in these paediatric tumour subtypes.

Although the decision for epidural analgesia performed under GA in a child cannot yet be mandated on oncologic grounds, we remain cognisant of its other well-studied benefits: ${ }^{(40)}$ superior analgesia (especially in thoracoabdominal surgeries), preservation of ventilatory mechanics and surgical stress reduction. It was also reassuring to observe no epidural-related complications in our database over the entire study period, consistent with that in large paediatric regional block databases. ${ }^{(41)}$ Hence, combined GA and epidural analgesia is a modality that we should definitely consider to facilitate functional recovery and timely return to intended oncologic adjuvant therapies in the short term ${ }^{(42)}$ while keeping the risk of recurrence to the minimum in the long term. After all, the global burden of cancer is not limited to adults, and the risk of relapse will plague the young for many more years to come.

\section{ACKNOWLEDEGEMENTS}

The authors thank A/Prof Tan Ah Moy and Ms Germaine Liew from the SCCR for providing the database from which our study population was identified.

\section{REFERENCES}

1. Tan AM, Ha C. Singapore Childhood Cancer Registry 2003-2007. Available at: https://www.nrdo.gov.sg/docs/librariesprovider3/Publications-Cancer/sccrmonograph-combined-v1-4.pdf?sfvrsn=0. Accessed October 1, 2019.

2. Snyder GL, Greenberg S. Effect of anaesthetic technique and other perioperative factors on cancer recurrence. Br J Anaesth 2010; 105:106-15.

3. Colvin LA, Fallon MT, Buggy DJ. Cancer biology, analgesics, and anaesthetics: is there a link? Br J Anaesth 2012; 109:140-3.

4. Kim R. Anaesthetic technique and cancer recurrence in oncologic surgery: unraveling the puzzle. Cancer Metastasis Rev 2017; 36:159-77.

5. Shakhar G, Ben-Eliyahu S. Potential prophylactic measures against postoperative immunosuppression: could they reduce recurrence rates in oncological patients? Ann Surg Oncol 2003; 10:972-92.

6. Maniwa Y, Okada M, Ishii N, Kiyooka K. Vascular endothelial growth factor increased by pulmonary surgery accelerates the growth of micrometastases in metastatic lung cancer. Chest 1998; 114:1668-75.

7. Holmgren L, O'Reilly MS, Folkman J. Dormancy of micrometastases: balanced proliferation and apoptosis in the presence of angiogenesis suppression. Nat Med 1995; 1:149-53.

8. Moudgil GC, Singal DP. Halothane and isoflurane enhance melanoma tumour metastasis in mice. Can J Anaesth 1997; 44:90-4.

9. Zhang T, Fan Y, Liu K, Wang Y. Effects of different general anaesthetic techniques on immune responses in patients undergoing surgery for tongue cancer. Anaesth Intensive Care 2014; 42:220-7.

10. Vallejo R, de Leon-Casasola O, Benyamin R. Opioid therapy and immunosuppression: a review. Am J Ther 2004; 11:354-65.

11. Gupta K, Kshirsagar S, Chang L, et al. Morphine stimulates angiogenesis by activating proangiogenic and survival-promoting signaling and promotes breast tumor growth. Cancer Res 2002; 62:4491-8.

12. Le-Wendling L, Nin O, Capdevila X. Cancer recurrence and regional anaesthesia: the theories, the data, and the future in outcomes. Pain Med 2016; 17:756-75.

13. Bar-Yosef S, Melamed R, Page GG, et al. Attenuation of the tumor-promoting effect of surgery by spinal blockade in rats. Anesthesiology 2001; 94:1066-73.

14. Wada H, Seki S, Takahashi T, et al. Combined spinal and general anaesthesia attenuates liver metastasis by preserving $\mathrm{TH} 1 / \mathrm{TH} 2$ cytokine balance. Anesthesiology 2007; 106:499-506.

15. Gupta A, Björnsson A, Fredriksson M, Hallböök O, Eintrei C. Reduction in mortality after epidural anaesthesia and analgesia in patients undergoing rectal but not colonic cancer surgery: a retrospective analysis of data from 655 patients in central Sweden. Br J Anaesth 2011; 107:164-70.

16. Exadaktylos AK, Buggy DJ, Moriarty DC, Mascha E, Sessler DI. Can anesthetic technique for primary breast cancer surgery affect recurrence or metastasis? Anaesthesiology 2006; 105:660-4.

17. Christopherson R, James KE, Tableman M, Marshall P, Johnson FE. Long-term survival after colon cancer surgery: a variation associated with choice of anaesthesia. Anesth Analg 2008; 107:325-32.

18. Cakmakkaya OS, Kolodzie K, Apfel CC, Pace NL. Anaesthetic techniques for risk of malignant tumor recurrence. Cochrane Database Syst Rev 2014; (11):CD008877.

19. Yardeni IZ, Beilin B, Mayburd E, Levinson Y, Bessler H. The effect of perioperative intravenous lidocaine on postoperative pain and immune function. Anesth Analg 2009; 109:1464-9.

20. Deegan CA, Murray D, Doran P, et al. Effect of anaesthetic technique on oestrogen receptor-negative breast cancer cell function in vitro. $\mathrm{Br} J$ Anaesth 2009; 103:685-90.

21. Page GG, Blakely WP, Ben-Eliyahu S. Evidence that postoperative pain is a mediator of the tumor-promoting effects of surgery in rats. Pain 2001; 90:191-9.

22. Biki B, Mascha E, Moriarty DC, et al. Anesthetic technique for radical prostatectomy surgery affects cancer recurrence: a retrospective analysis. Anesthesiology 2008; 109:180-7.

23. Holler JP, Ahlbrandt J, Burkhardt E, et al. Peridural analgesia may affect long-term survival in patients with colorectal cancer after surgery (PACO-RAS-Study): an analysis of a cancer registry. Ann Surg 2013; 258:989-93.

24. Call TR, Pace NL, Thorup DB, et al. Factors associated with improved survival after resection of pancreatic adenocarcinoma: a multivariable model. Anesthesiology 2015; 122:317-24.

25. Myles PS, Peyton P, Silbert B, et al; ANZCA Trials Group Investigators. Perioperative epidural analgesia for major abdominal surgery for cancer and recurrence-free survival: randomised trial. BMJ 2011; 342:d1491.

26. Weng $M$, Chen W, Hou W, et al. The effect of neuraxial anaesthesia on cancer recurrence and survival after cancer surgery: an updated meta-analysis. Oncotarget 2016; 7:15262-73.

27. Lin X, Wang YJ, Li Q, et al. Chronic high-dose morphine treatment promotes SH-SY5Y cell apoptosis via c-Jun N-terminal kinase-mediated activation of mitochondria-dependent pathway. FEBS J 2009; 276:2022-36.

28. Juneja R. Opioids and cancer recurrence. Curr Opin Support Palliat Care 2014; 8:91-101.

29. Olbrecht V, Siekerman M, Pinkard O, et al. Effect of common opioids and methylnaltrexone on pediatric tumor growth: differential expression of muopioid receptors in tumor lines. Available at: http://www5.pedsanesthesia.org/ meetings/2014winter/syllabus/submissions/aandp/walkarounds/PR2-123.pdf. Accessed October 1, 2019.

30. Yeager MP, Procopio MA, Deleo JA, et al. Intravenous fentanyl increases natural killer cell cytotoxicity and circulating CD16(+) lymphocytes in humans. Anesth Analg 2002; 94:94-9.

31. Cronin AJ, Aucutt-Walter NM, Budinetz T, et al. Low-dose remifentanil infusion does not impair natural killer cell function in healthy volunteers. Br J Anaesth 2003; 91:805-9.

32. Owusu-Agyemang $\mathrm{P}$, Hayes-Jordan A, Van Meter A, et al. Assessing the survival impact of perioperative opioid consumption in children and adolescents undergoing cytoreductive surgery with hyperthermic intraperitoneal chemotherapy. Paediatr Anaesth 2017; 27:648-56.

33. Lirk P, Berger R, Hollmann MW, Fiegl H. Lidocaine time- and dose-dependently demethylates deoxyribonucleic acid in breast cancer cell lines in vitro. $\mathrm{Br}$ J Anaesth 2012; 109:200-7.

34. Piegeler T, Votta-Velis EG, Liu G, et al. Antimetastatic potential of amidelinked local anesthetics: inhibition of lung adenocarcinoma cell migration and inflammatory Src signaling independent of sodium channel blockade. Anesthesiology 2012; 117:548-59.

35. Lucchinetti E, Awad AE, Rahman M, et al. Antiproliferative effects of local anesthetics on mesenchymal stem cells: potential implications for tumor spreading and wound healing. Anesthesiology 2012; 116:841-56.

36. Werdehausen R, Braun S, Fazeli S, et al. Lipophilicity but not stereospecificity is a major determinant of local anaesthetic-induced cytotoxicity in human T-lymphoma cells. Eur J Anaesthesiol 2012; 29:35-41.

37. Lüthy IA, Bruzzone A, Piñero CP, et al. Adrenoceptors: non-conventional target for breast cancer? Curr Med Chem 2009; 16:1850-62.

38. Forget $\mathrm{P}$, Collet $\mathrm{V}$, Lavand'homme $\mathrm{P}$, De Kock M. Does analgesia and condition influence immunity after surgery? Effects of fentanyl, ketamine and clonidine on natural killer activity at different ages. Eur J Anaesthesiol 2010; 27:233-40.

39. Allaouchiche B, Debon R, Goudable J, Chassard D, Duflo F. Oxidative stress status during exposure to propofol, sevoflurane and desflurane. Anesth Analg 2001; 93:981-5.

40. Bosenberg AT. Benefits of regional anaesthesia in children. Pediatr Anesth 2012; 22:10-8.

41. Ecoffey C, Lacroix F, Giaufré E, Orliaguet G, Courrèges P. Epidemiology and morbidity of regional anaesthesia in children: a follow-up one-year prospective survey of the French- Language Society of Paediatric Anaesthesiologists (ADARPEF). Paediatr Anaesth 2010; 20:1061-9.

42. Wigmore T, Gottumukkala V, Riedel B. Making the case for the subspecialty of onco-anaesthesia. Int Anesthesiol Clin 2016; 54:19-28. 


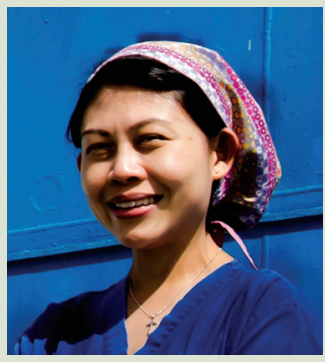

\section{About the First Author}

Dr Lee Shu Ying is a paediatric anaesthetist at KK Women's and Children's Hospital, Singapore. She completed her paediatric anaesthesia fellowship, with a focus on ultrasound-guided blocks at Alder Hey Children's Hospital, United Kingdom, and obtained the European Diploma in Regional Anaesthesia and Pain Therapy in 2012. She advocates for and teaches ultrasound-guided regional blocks in children. Her other research interests include perioperative temperature management. 